\title{
Otimização do Sorriso com Restaurações Diretas de Compósito Resinoso Nanoparticulado
}

\author{
Optimization of the Smile using Direct Resin Nanocomposite Restorations
}

\author{
Natália Azevedo ; Gabriel Galvão ; Valdeane Simone Cenci Nihiª Márcio Grama Hoeppnerc; Fábio Mitugui Nihia*
}

\author{
anniversidade Paranaense, Curso de Odontologia, PR, Brasil \\ • Universidade São Leopoldo Mandic, Programa de Pós-Graduação em Odontologia, SP, Brasil \\ 'Universidade Estadual de Londrina, Centro de Ciências da Saúde, PR, Brasil \\ *E-mail: fabionihi@unipar.br
}

Recebido: 01 de abril de 2014; Aceito: 01 de junho de 2014

\begin{abstract}
Resumo
Com o conhecimento das análises faciais, do sorriso e da harmonia dental, associados ao avanço das técnicas e materiais restauradores, tornouse possível solucionar alterações dentárias com a realização de restaurações diretas. Entre os tratamentos para restabelecer o equilíbrio do sorriso e a harmonia da beleza da face está o tratamento de fechamento de diastemas e a reanatomização por meio de restaurações diretas em dentes conóide, girovertidos e mal alinhados no arco dental. Baseados nas informações acima, o objetivo deste relato de caso foi apresentar a otimização do sorriso com restaurações diretas, utilizando resinas compostas. Paciente gênero feminino, 14 anos de idade, procurou a Universidade Paranaense - UNIPAR com queixa de espaços entre os dentes. Após avaliações clínicas e radiográficas e com o objetivo de melhorar de forma rápida e conservadora, foram realizadas restaurações diretas nos elementos 11 e 21 , modificando por meio do fechamento de diastema. Além disso, o proporcionamento restaurador do incisivo lateral conóide (12) e do canino (23), para suprir a agenesia do elemento 22 foi realizado. Foram utilizadas resinas compostas nanoparticuladas, associadas a um sistema adesivo convencional. O resultado alcançado demonstrou a possibilidade eficaz de tratamento restaurador direto, para devolver a harmonia do sorriso prejudicado por alterações de forma e posição, com a vantagem de ser uma técnica rápida, conservadora, reversível e de menor custo, quando comparado com restaurações indiretas.

Palavras-chave: Diastema. Resinas Compostas. Nanopartículas.
\end{abstract}

\begin{abstract}
Analysis for evaluating facial esthetics associated with the evolution of restorative materials and techniques allows solving dental changes by using direct restorations. Among the treatments to restore balance and harmony of the smile from the face of beauty is the treatment of diastema closure and reanatomização through direct restorations in conoid teeth, and misaligned girovertidos the dental arch. Based on the above, the purpose of this cstudy was to optimize a smile by direct restorations using composite resins. The patient, female, 14 years old, sought the Parana University (UNIPAR) complaining lack of space for her teeth. After clinical and radiographic evaluation, with the goal of improving quickly and conservatively, direct restorations in elements 11 and 21 were done to close diastema. Moreover, the proportioning restorer of the conoid lateral incisor (12) and canine (23) to supply the agenesis of element 22 was performed. Nanoparticulate composites were associated with conventional adhesive. The result demonstrated the possibility of effective direct restorative treatment to return the smile harmony harmed by changes in teeth shape and position, with the advantage of being a fast, conservative, reversible and less costly technique as compared to indirect restorations.
\end{abstract}

Keyword: Diastema. Composite Resins. Nanoparticles.

\section{Introdução}

O sorriso é de suma importância para o indivíduo, pois representa uma continuidade da estética facial, muito valorizada culturalmente no ocidente. Neste contexto, os dentes representam papel primordial, uma vez que completam o sorriso e a beleza da face. A presença de alterações como os diastemas, pode quebrar este equilíbrio, prejudicando a harmonia facial. Além de prejudicar a estética e a oclusão, influencia negativamente nas relações sociais do indivíduo ${ }^{1}$. Em uma pesquisa envolvendo adultos, constatou-se que a presença de amplos diastemas estava relacionada ao insucesso profissional e menor inteligência do indivíduo ${ }^{1}$. Desta forma, a Odontologia Estética pode trabalhar para tornar o sorriso mais belo, restabelecendo a estética facial e favorecer o convívio social².
A incidência dos diastemas pode variar em relação à idade, gênero e raça do indivíduo, ou seja, o fator genético atua diretamente na presença ou ausência do problema. Considerando o fator idade, a literatura afirma que $97 \%$ das crianças, aos cinco anos de idade, apresentam diastemas ${ }^{3}$, que diminuem grandemente entre nove e onze anos, até os 15 anos de idade. Outros estudos sobre a incidência de diastemas em crianças e adolescentes foram realizados ${ }^{3-5} \mathrm{e}$, em concordância entre os autores, foi observado que a maior incidência acometia meninos de todas as origens étnicas. Outro ponto em comum é o fator raça, no qual a incidência é maior para os melanodermas, seguido dos leucodermas e por último nos xantodermas. Analisando os fatores secundários que podem influenciar na incidência dos diastemas, é importante ressaltar 
que a etiologia do espaço entre os dentes está diretamente ligada à idade do paciente, bem como o tratamento proposto. Assim, pode-se considerar que os diastemas podem ser congênitos ou adquiridos, entretanto sua etiologia compreende vários fatores, tais como: presença de freio labial, crescimento e desenvolvimento anormal, ausência de dentes, presença de hábitos deletérios, iatrogenias, má oclusão e discrepâncias intermaxilares e presença de patologias ${ }^{6}$. Contudo, antes de determinar o tratamento mais indicado para solucionar a alteração, os fatores etiológicos devem ser considerados, pois o diagnóstico diferencial conduz a um tratamento mais individualizado e, portanto, mais efetivo ${ }^{2}$.

Dentre as técnicas utilizadas para realizar o fechamento de diastema estão o uso de aparelhos ortodônticos, uso de resina composta ou de cerâmica. Estas podem ser empregadas utilizando diferentes técnicas restauradoras/reabilitadoras, podendo ser empregadas técnicas diretas (fechamento de diastemas e facetas) ou indiretas, mais comumente utilizando cerâmicas. O emprego de resinas compostas, principalmente as de nanopartículas e de nanotecnologia, para o fechamento de diastemas apresenta vantagens em relação a outras técnicas, como a conservação da estrutura dental, reversibilidade do procedimento, menor custo ao paciente, menor tempo total do procedimento e facilidade de ser reparada, comparado com as restaurações indiretas ${ }^{7}$. Estudos ${ }^{8,9}$ têm demonstrado a eficácia destas técnicas, além do sucesso da técnica direta estar intimamente relacionado com o planejamento, habilidade do profissional, qualidade da resina composta, do sistema de união empregado e tipo de aparelho fotopolimerizador. Assim, o cuidado na seleção dos fatores e correta utilização estão relacionados à longevidade das restaurações estéticas diretas ${ }^{8}$.

$\mathrm{O}$ advento da nanotecnologia e a sua aplicação no desenvolvimento de compósitos restauradores possibilitaram aumentar a concentração da porção inorgânica à matriz resinosa pela redução do tamanho das partículas de carga. Consequentemente foram alcançadas melhorias nas propriedades mecânicas, bem como nas propriedades relacionadas com a estética, tais como a lisura superficial, reflexão de luz e estabilidade de cor, obtendo, como consequência, maior durabilidade da restauração ${ }^{2}$. Adicionalmente, os sistemas adesivos também ganharam com a nanotecnologia. A adição de tais partículas manométricas aos adesivos dentinários possibilitou uma maior resistência à interface adesiva, mesmo que esta fosse somente o substrato de esmalte ${ }^{8}$.
Diante do exposto, o objetivo deste estudo foi apresentar, por meio de um relato de caso clínico, o fechamento de diastemas em dentes anteriores e reanatomização de dentes conóides, utilizando resinas compostas nanoparticuladas pela técnica direta.

\section{Relato de Caso}

Paciente B.S., 14 anos de idade, gênero feminino, compareceu a clínica odontológica da Universidade Paranaense - UNIPAR - Umuarama, apresentando, como queixa principal, espaços no sorriso e ausência de dentes (Figura 1).

Figura 1: Aspecto inicial do sorriso evidenciando os diastemas pela presença do elemento 12 conóide e agenesia do 22 .

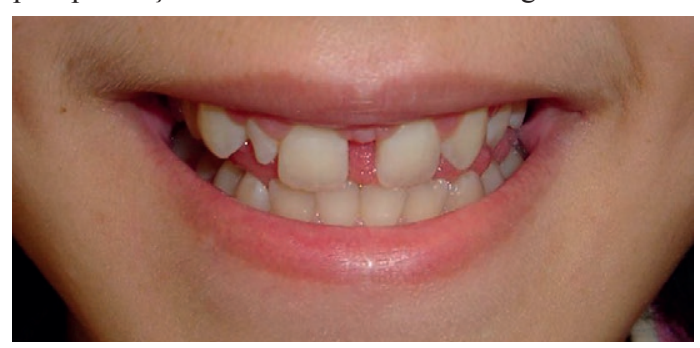

Após detalhada anamnese, exame clínico (Figuras 2 e 3) e exame radiográfico complementar, foi constatada presença de diastemas nos dentes anteriores devido à agenesia do elemento 22, alteração de forma congênita do elemento 12 (dente conóide). Após exposto as vantagens e desvantagens do tratamento e a assinatura do consentimento livre e esclarecido pela paciente e responsável, o tratamento escolhido foi a de restaurações diretas com resinas compostas, levando-se em consideração a efetividade, tempo de tratamento, custos e possibilidade de reversão do tratamento.

Figura 2: Vista frontal do caso inicial.

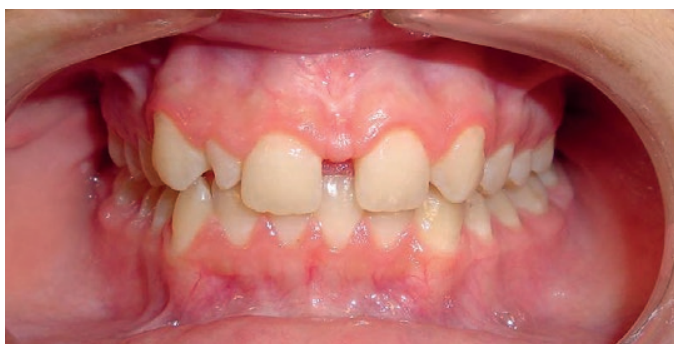

Figura 3: Testes clínicos de movimentos de lateralidade mandibular direita e esquerda (A e B).
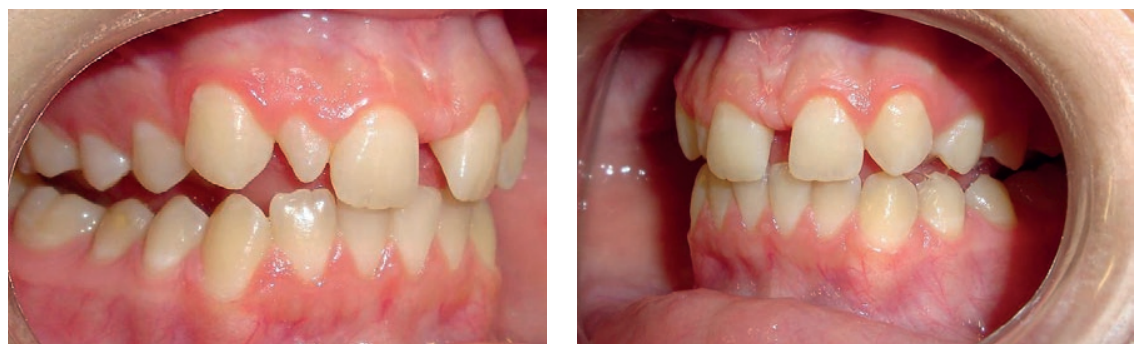
Para realização dos procedimentos restauradores, foi realizada a tomada de cor (A2 body + A1 enamel - Filtek Z350 XT - 3M ESPE, Saint Paul, Minnessota, USA) através da escala de cor disponibilizada pelo fabricante, seguido da realização do isolamento absoluto pela técnica modificada, também chamada de "canoa" (Figura 4 A-B).

Figura 4: Isolamento absoluto modificado ("tipo canoa"), demonstrando como o lençol permanece aderido à gengiva por meio de cola de cianocrilato.

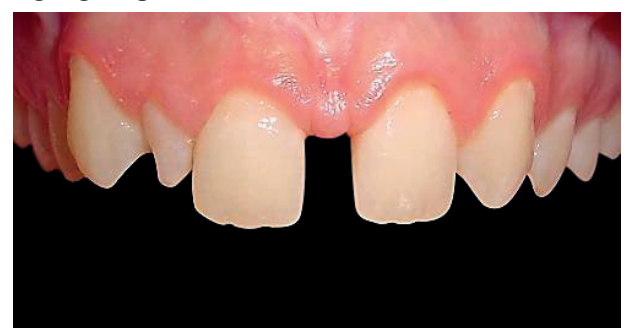

Com a finalidade de diminuir a presença da crista cérvicoincisal característica da anatomia da face vestibular dos caninos, e que não é tão marcada nos incisivos laterais, um slice ou plastia foi realizada com ponta diamantada de granulação fina $2135 \mathrm{~F}$ (KG SORENSEN, Cotia, São Paulo, Brasil) (Figura 5-A). O

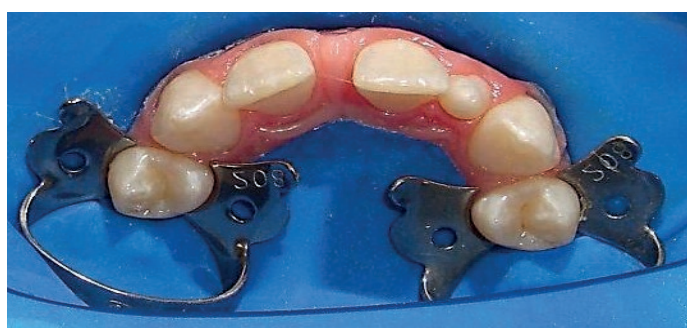

mesmo procedimento foi realizado para diminuir levemente a proeminência no centro da incisal, correspondente ao lóbulo central de desenvolvimento do elemento 23 (Figura 5-B) para então conseguir modificá-lo anatomicamente em um incisivo lateral com borda incisal plana e levemente inclinada para distal.

Figura 5: Desgastes mínimos (slice) realizados no elemento 23, necessários para proporcionamento e transformação do canino em incisivo lateral.

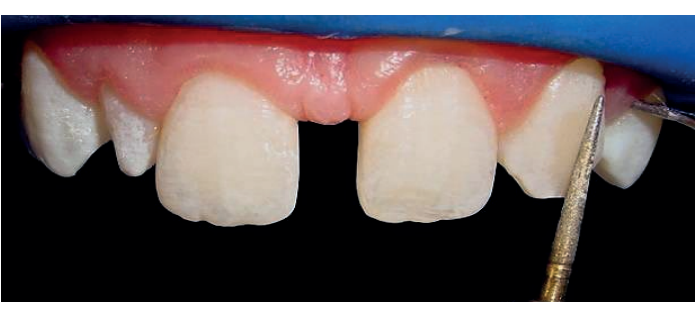

Os procedimentos da aplicação do sistema adesivo (ácido fosfórico a $37 \%$ e adesivo convencional de 2 passos operatórios) foram executados levando-se em consideração que as restaurações seriam realizadas sobre o substrato esmalte e, assim, considerados tempo de condicionamento de 30 segundos, seguidos de lavagem pelo mesmo tempo

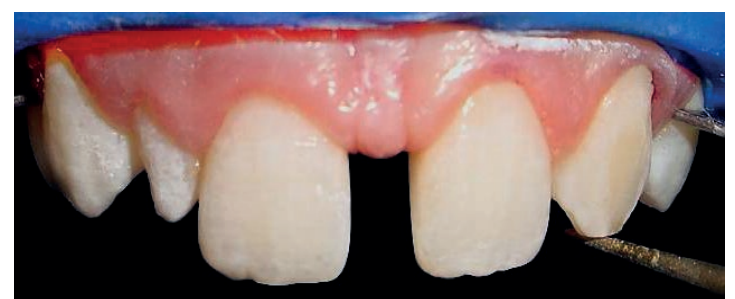

de condicionamento, e aplicação do adesivo Adper Single Bond 2 (3M ESPE, Saint Paul, Minnessota, USA), classificado como um adesivo convencional de $2 \operatorname{passos}^{10}$ que apresenta como solvente etanol + água, com a presença de partículas de carga nanoparticuladas (Figuras 6 A, B e C).

Figura 6: Procedimentos de aplicação do sistema adesivo sobre a superfície do esmalte e fotoativação.
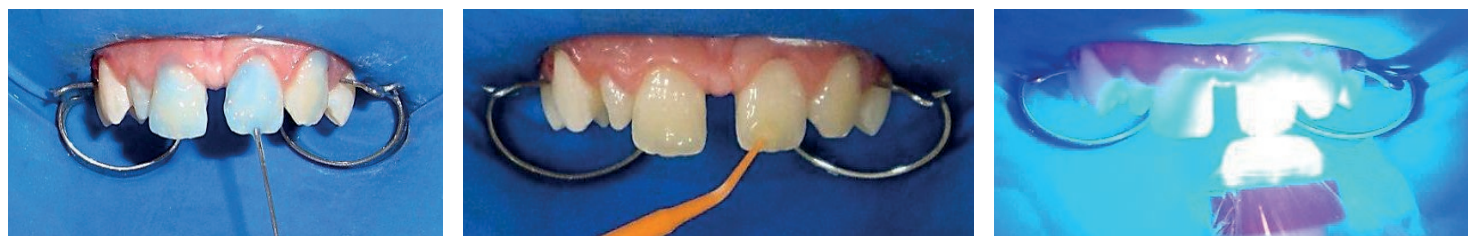

Para a técnica restauradora, os incrementos de resina composta foram inseridos, respeitando a técnica de estratificação (Figuras 7 e 8). Isto consiste na inserção das porções, restabelecendo, primeiro, o formato dentinário, seguindo os lóbulos de desenvolvimento, seguido de uma fina camada para reprodução da porção que corresponde ao esmalte. Para tanto, foram utilizadas as resinas de corpo
(Body), já que apresentam uma translucidez intermediária entre a de dentina e a de esmalte, seguidas das resinas de esmalte (Enamel), cuja translucidez é acentuada. Para cada incremento de no máximo $2 \mathrm{~mm}$ de espessura, seguiu-se a fotoativação com aparelho de LED (Radii Cal, SDI, São Paulo, São Paulo, Brasil) com densidade de energia de $800 \mathrm{~mW} / \mathrm{cm}^{2}$, por 40 segundos. 
Figura 7: Inserções de incrementos de resina composta para o fechamento dos diastemas

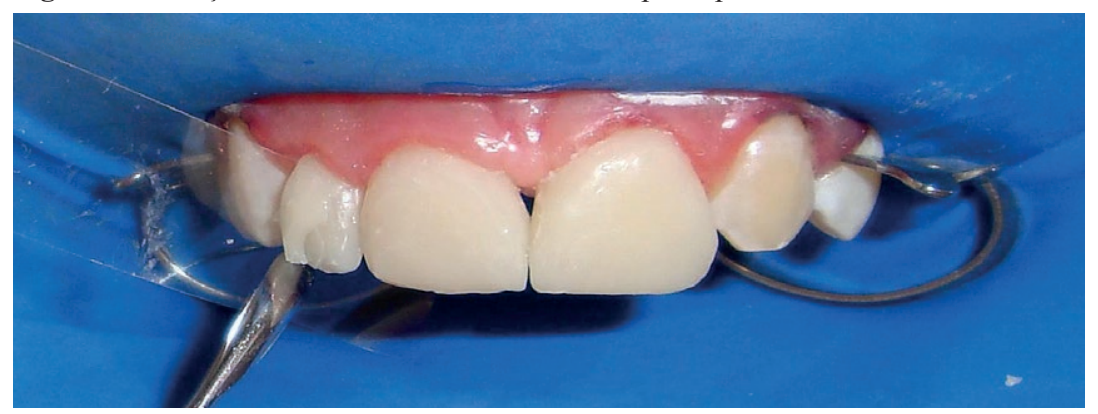

Figura 8: Proporcionamento e transformação do elemento canino (elemento 23) em incisivo lateral, fechando o diastema.

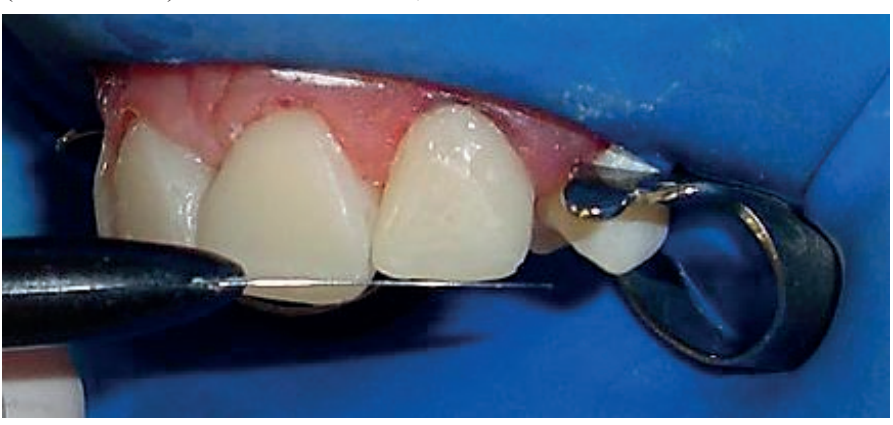

$\mathrm{Na}$ mesma sessão, foram realizados os procedimentos de acabamento e texturas com pontas diamantadas da série F (granulação fina), as microtexturas (Figura 9-A) e polimento com sequencial de discos de lixa (Sof Lex
Pop On - 3M ESPE, Saint Paul, Minnessota, USA), com posterior disco de feltro e pasta diamantada (Diamond Excel - FGM, Joinville, Santa Catarina, Brasil) (Figura 9-B).

Figura 9: Procedimentos de acabamento e polimento realizados com pontas diamantadas e disco de feltro + pasta diamantada.

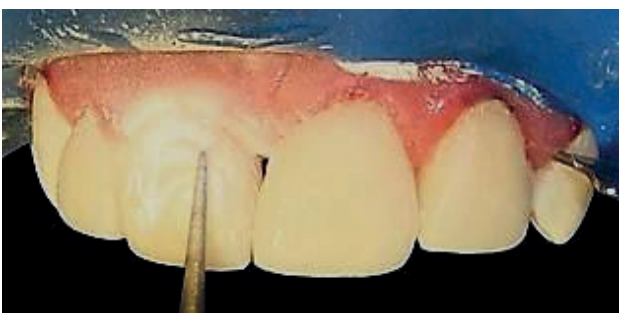

Os contatos oclusais foram checados para certificar que nenhuma interferência permanecesse, já que a guia anterior dos movimentos mandibulares de protrusão fora modificada pelo acréscimo de resina composta (Figura 10).

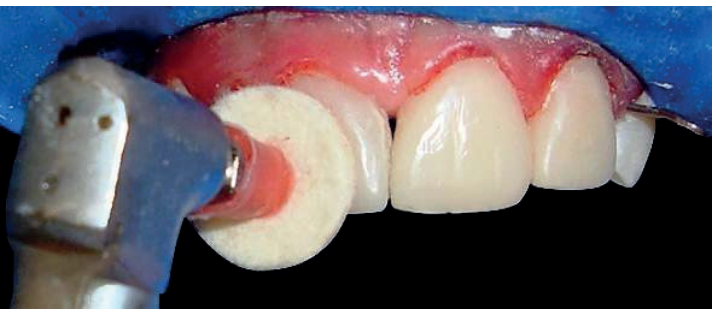

Nas Figuras 11 a 13 pode-se visualizar o aspecto final das restaurações e do sorriso com término imediato após as restaurações e a reanatomização dos dentes ântero superiores.

Figura 11: Aspecto final

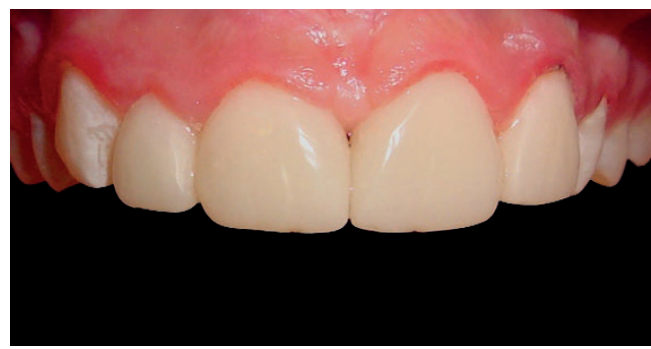


Figura 12: Aspecto final

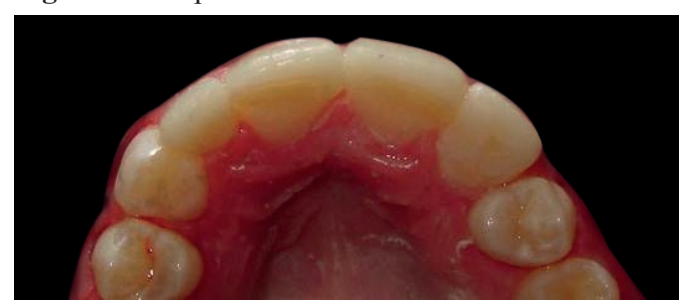

Figura 13: Sorriso da paciente ao final das restaurações.

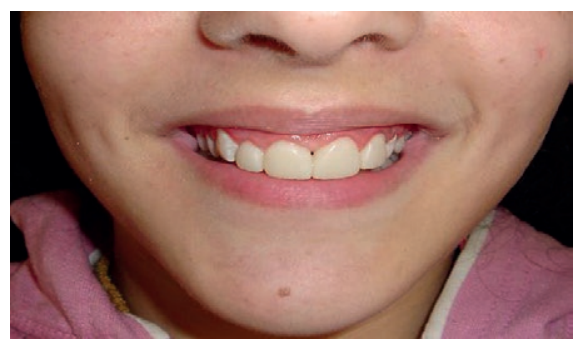

\section{Discussão}

A constante busca por tratamentos estéticos contribuiu com o desenvolvimento de técnicas e métodos restauradores inovadores para solucionar alterações nos dentes e no sorriso. Frente à presença de pacientes com diastemas, principalmente entre os elementos ântero superiores, e ao desafio de conduzir um bom tratamento para um problema bastante frequente, um correto diagnóstico aliado à determinação do fator etiológico e um exame clínico apurado parece ser de suma importância. No caso clínico apresentado, a paciente procurou atendimento odontológico como queixa principal a estética dos dentes, uma vez que o seu convívio social, mesmo que com pouca idade, já era prejudicado, inclusive tendo influências negativas em seu desempenho escolar. Considerando estes aspectos somados, demonstrou a necessidade de intervenção imediata, mesmo que precoce, na estética do sorriso. Pelo sorriso representar um fator de grande importância no contexto social, Jacobson ${ }^{11}$ afirma que o sucesso profissional e a beleza da face são um facilitador na atração de amigos, influenciando positivamente o convívio social. Da mesma forma, Cunningham ${ }^{12}$ e Mondelli13 ${ }^{13}$ também afirmam que o sorriso pode influenciar grandemente na autoestima do indivíduo e, assim, favorecer o sucesso profissional. Uma explicação para tal fato é de que, psicologicamente, indivíduos se sentem mais motivados e, portanto, se tornam seres mais atraentes ${ }^{11}$. No caso apresentado, além da presença de diastemas (13 ao 23) e agenesia (22), houve também incidência de dente conóide (12), aumentando ainda mais a necessidade de tratamento restaurador rápido e seguro. Somado a isto, o ônus dispensado para a realização dos demais tratamentos influenciou na escolha da paciente e de seu responsável, dentre as opções apresentadas.

Como já mencionado, a etiologia dos diastemas podem ser desde simples discrepâncias entre a forma e tamanho inerente a um único elemento dental, também pela presença de inserções musculares (freios), até casos mais graves de alterações ósseas de maxila e mandíbula. A etiologia dos diastemas diagnosticada no presente caso clínico foi a ausência de dentes, ou seja, agenesia do elemento 22 e a presença de alteração de forma diminuída de um dente conóide (12). A agenesia, ou ausência congênita do germe dental, pode ser encontrada na dentadura permanente numa prevalência que pode variar de 0,8 a $4,25 \%{ }^{14,15}$. Ainda não estão claros os mecanismos para o acometimento desta alteração e também não há um consenso entre os autores sobre as causas dessas ocorrências. Portanto, a literatura assim descreve como sendo uma falha correlacionada com o desenvolvimento maxilar ${ }^{16}$. Já o dente conóide é caracterizado por uma alteração congênita de desenvolvimento do germe dentário, relacionada com o tamanho e forma, que ocorre na fase de aposição ou deposição dos tecidos dentários. É uma microdontia, cuja raiz se apresenta de tamanho normal, e sobre ela, a presença de uma coroa de menor proporção e em forma de cone ${ }^{17}$. Laterais conóides acometem indivíduos numa prevalência de $0,5 \%$ a $3 \%$ e têm origem genética autossômica dominante, geralmente associada com outras hipodontias, como descrito por Izgi ${ }^{18}$. No caso demonstrado, a paciente apresentou somente o elemento 12 com microdontia, sem ocorrer em nenhum outro elemento. Entretanto, assim como relatado anteriormente por Izgi ${ }^{18}$, houve o acometimento de outra anomalia no elemento 22 (agenesia), associada ao dente conóide.

A odontologia cosmética não se resume à restauração da forma e da função dos elementos dentais, mas também tem a capacidade de criar um novo sorriso que se adapte ao estilo de vida do paciente. Desta forma, optou-se pela restauração com resinas compostas diretas, melhorando a estética e fechando os diastemas presentes. Para isso, se faz necessários materiais com elevada resistência à fratura, devido à borda incisal dos dentes anteriores ser uma área de grande concentração de forças, resultante dos movimentos bordejantes $^{19}$. Optou-se pelas resinas compostas de nanopartículas por apresentarem elevada concentração de partículas de carga inorgânica, chegando a $75 \%$ em peso, com um módulo de elasticidade de $10.500 \mathrm{MPa}^{20}$. Além disso, o fato destas partículas nanométricas estarem inseridas de forma livre no interior da matriz orgânica e não formando aglomerados, classifica esta resina como de nanopartícula de fato, trazendo melhorias às propriedades estéticas da restauração. A matriz orgânica e seus componentes, bem como o tamanho de suas partículas de carga, exercem grande influência na superfície da resina composta. Quanto maior o tamanho das partículas de carga, maior será a rugosidade, dificultando o polimento e possibilitando maior manchamento ${ }^{7}$. A porção inorgânica representada pelas partículas de carga tem um papel preponderante neste aspecto mecânico e estético da resina composta. O problema de aumentar o tamanho das partículas é que elas tendem a 
se soltar com maior facilidade e causar maior manchamento pela maior exposição da matriz orgânica que se apresenta hidrofílica, ou seja, uma afinidade por água. Sabe-se também que superfícies com maior rugosidade superficial tendem a um maior acúmulo de biofilme dental, o que facilita as lesões cariosas secundárias ${ }^{21}$. Assim, dentre as grandes vantagens que estas resinas apresentam estão a elevada lisura superficial, brilho ${ }^{22}$ e estabilidade de $\operatorname{cor}^{23}$, que fazem desta resina composta uma excelente opção restauradora para dentes anteriores. Juntamente com partículas dispersas, apresentam nanoclusters ou nanoaglomerados, que aumentam as propriedades mecânicas da resina por elevarem a proporção de partículas inorgânicas em relação à matriz orgânica. Uma explicação para maior durabilidade das restaurações com resinas compostas nanoparticuladas é que quanto maior a concentração de partículas e maior o módulo de elasticidade, menor é a contração de polimerização livre $^{24}$. Conhecendo o fenômeno da polimerização dos compósitos, sabe-se que este aspecto ainda é um desafio para as restaurações adesivas, pois a polimerização é uma característica inerente a este tipo de material e que não pode ser totalmente evitada, mas controlada ou diminuída. Alguns autores, entretanto, afirmam que se a resina composta estiver aderida às paredes de uma cavidade, causando resistência do incremento de material, as forças geradas nas resinas de nanopartículas também gerarão forças consideravelmente altas $^{25}$. Analisando todos os aspectos descritos, a opção para solucionar o caso clínico foi o uso da resina de nanopartículas Z350 XT (3M ESPE, Saint Paul, Minnessota, USA).

Existe grande preocupação para realização de restaurações adesivas, principalmente quanto a sua durabilidade. Neste sentido, uma correta hibridização dos substratos dentários (esmalte e dentina) deve ser criteriosamente realizada. Diferentemente do esmalte, a dentina apresenta uma constituição mais complexa, incluindo a presença de água e componentes orgânicos ${ }^{26}$, tornando as restaurações adesivas um desafio ainda maior. A literatura é ampla neste aspecto e diversas pesquisas in vitro ${ }^{27}$ e in vivo ${ }^{28}$ demonstram que quando as margens de restaurações adesivas se localizarem exclusivamente em dentina, a degradação da interface dente - restauração é ainda maior, quando comparada às de restauração com limites em esmalte, ao longo do tempo. No presente caso clínico, todas as restaurações, incluindo todos os elementos restaurados, foram confeccionadas sobre esmalte, concordando com a literatura e objetivando uma maior durabilidade do tratamento restaurador realizado. Uma vez que o substrato foi o esmalte, e por este ser quase totalmente homogêneo em sua composição ( $97 \%$ de conteúdo mineral), a opção foi a de um de sistema adesivo simplificado, convencional de 2 passos $^{10}$. Caso ocorra a necessidade de restaurações com limites em dentina, a recomendação é a utilização de sistemas adesivos que apresentem monômeros menos hidrofílicos ${ }^{29}$, com menor concentração de solventes, com pH menos ácidos, e portanto, os menos simplificados ${ }^{30,31}$.
A justificativa é de que quanto maior o $\mathrm{pH}$ e maior a concentração de solventes, maior será a hidrofilia da interface adesiva, podendo favorecer o processo de degradação adesiva, pela umidade dentinária. Assim, sistemas adesivos que apresentam monômero hidrofóbico com o último passo da hibridização formam uma barreira menos permeável ao processo de hidrólise pela umidade dentinária, mesmo após a polimerização ${ }^{31}$.

Para restaurar e reconstruir dentes anteriores, uma técnica preconizada recentemente é o uso de enceramento de diagnóstico, associado ao uso de matriz ou barreira de silicone $^{32}$. O uso de barreira de silicone obtida a partir da moldagem da arcada pode propiciar uma maior facilidade na reconstrução de elementos fraturados ou extensas reconstruções, ao passo que envolve um maior número de sessões clínicas, devido à realização de moldagem e incrementos, para finalização do tratamento restaurador. Por outro lado, o emprego da tira de poliéster diretamente sobre o dente, como no caso apresentado, permite a reconstrução imediata dos dentes, mas a sua efetividade fica condicionada à habilidade do profissional em manusear sobre a tira, a resina composta $^{33}$.

É importante ressaltar que vários tratamentos já foram propostos, dependendo da sua etiologia desde frenectomias, tratamentos ortodônticos, até tratamentos mais radicais como osteotomias e exodontias. Entretanto nenhum método pode ser eleito como prioridade para solucionar os diastemas. O êxito do tratamento odontológico será alcançado quando se pratica de forma multidisciplinar, aplicando os conceitos das diferentes especialidades nas diferentes etiologias.

\section{Conclusão}

A ciência e a arte com resinas compostas estão cada vez mais acessíveis ao clínico. Com o avanço na composição dos materiais e com o desenvolvimento de técnicas, possibilitam ao clínico resinas mais resistentes, mais estéticas e mais duradouras. O caso clínico apresentado demonstra a possibilidade da reprodução artística dos dentes naturais, utilizando resinas compostas de nanopartículas para solucionar diastemas causados por agenesias e alterações de forma. Pela dedicação e excelência estética, foi possível alcançar êxito no tratamento proposto de forma rápida, segura e principalmente reversível.

\section{Referências}

1. Kerosuo H, Hausen H, Laine T, Shaw WC. The influence of incisal malocclusion on the social attractiveness of young adults in Finland. Eur J Orthod 1995;17(6):505-12.

2. Furuse AY, Franco EJ, Mondelli J. Esthetic and functional restoration for an anterior open occlusal relationship with multiple diastemata: a multidisciplinary approach. J Prosthet Dent 2008;99(2):91-4.

3. Moura C, Cavalcanti AL, Gusmão ES, de Souza Coelho Soares R, Cavalcante Moura FT, Hordonho Santillo PM. Negative self-perception of smile associated with malocclusions among Brazilian adolescents. Eur J Orthod 2012 Apr 24. 
4. Bhardwaj VK, Veeresha KL, Sharma KR. Prevalence of malocclusion and orthodontic treatment needs among 16 and 17 year-old school-going children in Shimla city, Himachal Pradesh. Indian J Dent Res 2011;22(4):556-60.

5. Martins MG, Lima KC. Prevalence of malocclusions in 10- to 12-year-old schoolchildren in Ceará, Brazil. Oral Health Prev Dent 2009;7(3):217-23.

6. Huang WJ, Creath CJ. The midline diastema: a review of its etiology and treatment. Pediatr Dent 1995;17(3):171-9.

7. Heymann HO, Hershey HG. Use composite resin for restorative and orthodontic correction of anterior interdental spacing. J Prosthet Dent 1985;53(6):766-71.

8. De Araujo Junior EM, Fortkamp S, Baratieri LN. Closure of diastema and gingival recontouring using direct adhesive restorations: a case report. J Esthet Restor Dent 2009;21(4):229-40.

9. Rickman LJ, Padipatvuthikul P, Chee B. Clinical applications of preheated hybrid resin composite. Br Dent $\mathrm{J}$ 2011;211(2):63-7.

10. Van Meerbeek B, Perdigão J, Lambrechts P, Vanherle G. The clinical performance of adhesives. J Dent 1998;26(1):1-20.

11. Jacobson A. Psychological aspects of dento-facial esthetics and orthognatic surgery. Angle Orthod 1984;54;18-35

12. Cunningham SJ, Feinmann C. Psychological assessment of patients requesting orthognathic surgery and the relevance of body dysmorphic disorder. Br J Orthod 1998;25(4):293-8.

13. Mondelli J. Estética e cosmética em clínica integrada restauradora. São Paulo: Quintessence; 2003.

14. Tavajohi-Kermani H, Kapur R, Sciote JJ. Tooth agenesis and craniofacial morphology in na orthodontic population. Am J Orthod Dentofacial Orthop 2002;122(1):39-47.

15. Harris EF, Clark LL. Hypodontia: an epidemiologic study of American black and white people. Am J Orthod Dentofacial Orthop 2008;134(6):761-7.

16. Pinho T, Pollmann C, Calheiro-Lobo MJ, Souza A, Lemos C. Craniofacial repercussions in maxillary lateral incisors agenesis. Int Orthod 2011;9(3):274-85.

17. Pinho T, Maciel P, Lemos C, Sousa A. Familial aggregation of maxillary lateral incisor agenesis. J Dent Res 2010;89(6):621-5.

18. Izgi AD, Ayna E. Direct restorative treatment of peg-shaped maxillary lateral incisors with resin composite: a clinical report. J Prosthet Dent 2005;93(6):526-9.

19. Hobson RS, McCabe JF, Hogg SD. Bond strength to surface enamel for different tooth types. Dent Mater 2001;17(2):184-9.

20. Pereira SG, Osorio R, Toledano M, Nunes TG. Evaluation of two Bis-GMA analogues as potential monomer diluents to improve the mechanical properties of light-cured composite resins. Dent Mater 2005;21(9):823-30.
21. Kim KH, Ong JL, Okuno O. The effect of filler loading and morphology on the mechanical properties of contemporary composites. J Prosthet Dent 2002;87(6):642-9.

22. Takanashi E, Kishikawa R, Ikeda M, Inai N, Otsuki M, Foxton RM, et al. Influence of abrasive particle size on surface properties of flowable composites. Dent Mater J 2008;27(6):780-6.

23. Attar N. The effect of finishing and polishing procedures on the surface roughness of composite resin materials. J Contemp Dent Pract 2007;8(1):27-35.

24. Aguiar FH, Georgetto MH, Soares GP, Catelan A, Dos Santos $\mathrm{PH}$, Ambrosano GM, et al. Effect of different light-curing modes on degree of conversion, staining susceptibility and stain's retention using different beverages in a nanofilled composite resin. J Esthet Restor Dent 2011;23(2):106-14.

25. Mine A, De Munck J, Cardoso MV, Van Landuyt KL, Poitevin A, Kuboki T, et al. Effect of low-shrinking composite on the bonding effectiveness of two adhesives in occlusal Class-I cavities. Dent Mater J 2012;31(3):418-26.

26. Tjäderhane L, Nascimento FD, Breschi L, Mazzoni A, Tersariol IL, Geraldeli S, et al. Optimizing dentin bond durability: Control of collagen degradation by matrix metalloproteinases and cysteine cathepsins. Dent Mater 2012 Aug 16.

27. Geerts S, Bolette A, Seidel L, Guéders A. An in vitro evaluation of leakage of two etch and rinse and two self-etch adhesives after thermocycling. Int J Dent 2012;22.

28. Alptekin T, Ozer F, Unlu N, Cobanoglu N, Blatz MB. In vivo and in vitro evaluations of microleakage around Class I amalgam and composite restorations. Oper Dent 2010;35(6):641-8.

29. Bail M, Malacarne-Zanon J, Silva SM, Anauate-Netto A, Nascimento FD, Amore R, et al. Effect of air-drying on the solvent evaporation, degree of conversion and water sorption/ solubility of dental adhesive models. J Mater Sci Mater Med 2012;23(3):629-38.

30. Faria-E-Silva AL, Araújo JE, Rocha GP, Oliveira AD, Moraes RR. Solvent content and dentin bond strengths using waterwet, ethanol-wet and deproteinization bonding techniques. Acta Odontol Scand 2012;20.

31. Malacarne J, Carvalho RM, Goes MF, Svizero N, Pashley DH, Tay FR, et al. Water sorption/solubility of dental adhesive resins. Dent Mater 2006;22(10):973-80.

32. Kulkarni VK, Ragavendra TR, Deshmukh J, Vanka A, Duddu MK, Patil AK. Endodontic treatment and esthetic management of a primary double tooth with direct composite using silicone buildup guide. Contemp Clin Dent 2012;3(1):S92-5.

33. Souza-Junior EJ, Borges BCD, Bertoldo CES, Lovadino JR, Aguiar FHB, Paulillo LAMS. Restauração estética direta de dente anterior fraturado: relato de caso clínico. Rev Dental Press Estét 2010;7(4):42-51. 